\title{
Various effects of hepatoma-derived growth factor on cell growth, migration and invasion of breast cancer and prostate cancer cells
}

\author{
ZHENYING GUO ${ }^{1}$, YUANZHENG HE $^{2}$, SHUANGSHUANG WANG ${ }^{1}$, AIXIA ZHANG ${ }^{1}$, \\ PING $\mathrm{ZHAO}^{2}, \mathrm{CHONGFENG} \mathrm{GAO}^{2}$ and BRIAN CAO ${ }^{1,2}$ \\ ${ }^{1}$ Nanjing Medical University, Department of Pathology, Nanjing 210029, P.R. China; \\ ${ }^{2}$ Van Andel Research Institute, Grand Rapids, MI 49503, USA
}

Received March 18, 2011; Accepted April 21, 2011

DOI: 10.3892/or.2011.1295

\begin{abstract}
Hepatoma-derived growth factor (HDGF) has been implicated in the growth and metastasis of various types of human cancer, but the role of HDGF expression in prostate cancer or breast cancer has not been documented. To assess the role of HDGF in the proliferation, migration and invasion by prostate and breast cancer cells, HDGF expression in DU145 and MCF7 cells was knocked down using siRNA, and the effect of such knockdown was assessed by MTS and $\left[{ }^{3} \mathrm{H}\right]$-thymidine incorporation Transwell assays. Moreover, we identified differentially expressed genes that might mediate the HDGF-induced cellular effects. Our results demonstrate that down-regulation of HDGF expression significantly reduces the proliferation of both DU145 and MCF7 cells. However, down-regulation of HDGF expression in DU145 inhibited cell migration and invasion, but in MCF7 cells it stimulated cell migration and invasion. This differential effect might result from the differential induction of PIK3RI or SERPINE1 in the two cell lines upon HDGF-siRNA treatment. In conclusion, HDGF may participate in the pathogenesis of prostate and breast cancer by promoting cell growth and it may be a therapeutic target for these cancers.
\end{abstract}

\section{Introduction}

Hepatoma-derived growth factor (HDGF), a heparin-binding growth factor, stimulates the growth of $\mathrm{HuH}-7$ cells in serumfree chemically defined medium (1-3). HDGF contains a well-conserved $\mathrm{N}$-terminal amino acid sequence called the HATH region $(4,5)$, which is homologous to the amino terminus of HDGF. HDGF contains nuclear localization signals, and its

Correspondence to: Dr Brian Cao or Dr Chongfeng Gao, Van Andel Institute, 333 Bostwick Ave. NE, Grand Rapids, MI 49503, USA

E-mail: brian.cao@vai.org

E-mail: chongfeng.gao@vai.org

Key words: hepatoma-derived growth factor, prostate cancer, breast cancer, small interfering RNA nuclear translocation is essential for the induction of cell growth activity (6).

HDGF is highly expressed in tumor cells. It has a mitogenic function in cells, such as human hepatocellular carcinoma cells, fibroblasts, endothelial cells, vascular smooth muscle cells, and fetal hepatocytes (7-10). Overexpression of HDGF in lung cancer is a prognostic factor for its metastatic potential (11-13). Down-regulation of HDGF in non-small cell lung cancer cells by chemically synthesized shRNA suppresses cell growth and invasion in vitro (14), further supporting a role for HDGF in the development and progression of lung cancer. In pancreatic cancer, patients that had $<90 \%$ of cancer cells positively stained for nuclear HDGF showed a significantly higher 5 -year survival rate $(37.0 \%)$ than those with a $>90 \%$ nuclear cell staining $(6.8 \%$; $\mathrm{P}=0.023)$. No significant difference was observed in terms of the cytoplasmic HDGF classification between patients with $<90 \%$ and those with $>90 \%$ HDGF positively stained cells in the cytoplasm, which suggests that nuclear HDGF is an independent prognostic factor for pancreatic ductal carcinoma (15).

In a recent study, we found that HDGF is frequently overexpressed in breast and prostate cancer. To elucidate the mechanism of HDGF-mediated aggressiveness in breast and prostate cancer cells, we down-regulated HDGF expression in these cells using small interfering RNA (siRNA) technology and studied the effects of the down-regulation on cell proliferation, migration and invasion. Our results suggest that HDGF is involved in anchorage-independent growth and invasion by breast and prostate cancer cells. These qualities may contribute to the HDGF-associated aggressive biological behavior of breast and prostate cancers.

\section{Materials and methods}

Cell cultures. The human breast cancer cell line MCF7 and the human prostate cancer cell line DU145 were obtained from ATCC. MCF7 cells were cultured in Dulbecco's modified essential medium (DMEM) with $10 \%$ fetal bovine serum (FBS) (Invitrogen, Carlsbad, CA, USA) at $37^{\circ} \mathrm{C}$ with $5 \% \mathrm{CO}_{2}$. DU145 cells were cultured in RPMI-1640 (Invitrogen) with $10 \% \mathrm{FBS}$ at $37^{\circ} \mathrm{C}$ with $5 \% \mathrm{CO}_{2}$. 
Short interference RNA knockdown. The functional role of HDGF was assessed using siRNAs designed and synthesized for HDGF knockdown. The two siRNA sequences, purchased from Sigma, targeted three HDGF variants NM_001126050.1, NM_001126051.1 and NM_004494.2. SiRNA-1 (S1) was a duplex of 5'-GCCAUGUCUUCUCCCUGGA[dT][dT] and 5'-UCCAGGGAGAAGACAUGGC[dT][dT]. SiRNA-2 (S2) was a duplex of 5'-CUGACACCCUUCUCCUGGA[dT][dT] and 5'-AGUGGGAGAAGGGUGUCAG[dT][dT]. The control siRNA was purchased from Qiagen. In vitro transfections were performed using Lipofectamine RNAiMax (Invitrogen) following the manufacturer's protocols.

Transfection. Gene knockdown was achieved in DU145 and MCF7 cells using reverse transfection. Briefly, we diluted $30 \mathrm{pg}$ siRNA or negative control siRNA in $500 \mu \mathrm{l} /$ well of Opti-MEM I medium without serum in a 6-well plate. We then added $5 \mu \mathrm{l}$ RNAiMax to each well containing the diluted RNAi molecules, mixed gently and incubated for $15 \mathrm{~min}$ at room temperature. We next added $5 \times 10^{4}$ cells/well in $2.5 \mathrm{ml}$ serum-free medium on a 6 -well plate and mixed gently by rocking the plate back and forth. This gave a final volume of $3 \mathrm{ml}$ and a final RNA concentration of $10 \mathrm{nM}$. We added the complex to the corresponding wells, and cells were incubated for $72 \mathrm{~h}$ at $37^{\circ} \mathrm{C}$ with $5 \% \mathrm{CO}_{2}$ under standard conditions before being assayed.

Western blotting. Cells were washed twice with cold phosphatebuffered saline (PBS) and lysed on ice in RIPA buffer with protease inhibitors (Roche Diagnostics, Germany). Cell lysates (20 $\mu \mathrm{g} /$ lane) were loaded on a $10 \%$ SDS-polyacrylamide gel (SDS-PAGE), separated by electrophoresis and transferred to a nitrocellulose membrane. The membrane was blocked with blocking buffer (LI-COR, USA) for $1 \mathrm{~h}$ at room temperature, followed by incubation with purified mouse anti-human HDGF mAb (16) $(1 / 1,000$ from $2 \mathrm{mg} / \mathrm{ml})$ at $4^{\circ} \mathrm{C}$ overnight, and then followed by the goat anti-mouse IRDye $680(1 / 15,000$ from $1 \mathrm{mg} / \mathrm{ml}$; LI-COR) or the goat anti-rabbit IRDye 680 (1/15,000 from $1 \mathrm{mg} / \mathrm{ml}$; LI-COR) as a secondary antibody and fluorescence agent. The immune-reactive band was detected by the Odyssey infrared imaging system. $\beta$-tubulin was used as a protein loading control. Labeling was performed with a horse anti-mouse monoclonal $\beta$-tubulin antibody in (1:10,000 from $2 \mathrm{mg} / \mathrm{ml})$.

Cell proliferation assay. Cells transfected with siRNA were plated in 96-well plates at a density of $2 \times 10^{3}$ cells/well in medium containing $10 \%$ FBS and incubated for 3 days. The medium was then changed to serum-free medium for half of the wells. The number of cells was determined at 5 days after transfection using an MTS assay (Cell Titer 96 Cell Proliferation Assay, Promega, USA) according to the manufacturer's instructions. All experiments were repeated more than twice. Western blots were run to make sure cells were actually transfected in every experiment.

Thymidine incorporation assays. Cells transfected with siRNA for 3 days were distributed into 96-well plates ( $2 \times 10^{3}$ cells/well). The cells were then cultured for $48 \mathrm{~h}$ either under normal conditions or under starvation conditions in media without FBS. $\left[{ }^{3} \mathrm{H}\right]$-thymidine $(1 \mu \mathrm{Ci} / \mathrm{ml})$ (Amersham Biosciences, Pittsburgh, PA) was added to each well $4 \mathrm{~h}$ before analysis. Cells were washed with PBS, precipitated with chilled $10 \%$ trichloroacetic acid, and solubilized in $50 \mu \mathrm{l}$ of lysis buffer (0.02 M NaOH/0.1\% SDS). Cell lysates were suspended in $150 \mu \mathrm{l}$ of scintillation mixture (Packard Bioscience), and incorporation of $\left[{ }^{3} \mathrm{H}\right]$-thymidine was measured by liquid scintillation counting (MicroBeta TriLux, Perkin-Elmer, USA).

Anchorage-independent growth assay. Twenty-four hours after transfection with siRNA, 20,000 cells in $2 \mathrm{ml}$ of $0.35 \%$ agarose with DMEM were plated in each well of 6-well tissue culture plates on the top of the existing $0.7 \%$ bottom agarose, in triplicate for each treatment condition. The plates were covered with $2 \mathrm{ml}$ of medium with $10 \% \mathrm{FBS}$ and incubated at $37^{\circ} \mathrm{C}$ in a $5 \% \mathrm{CO}_{2}$ incubator for three weeks. The covering medium was replaced every three days. At the end of three weeks, cell colonies $>0.1 \mathrm{~mm}$ in diameter were counted under a microscopic field at $\mathrm{x} 40$ magnification. Means were based on the number of colonies from triplicate wells for each treatment condition and were analyzed using the two-sided Student's t-test.

In vitro migration and invasion assay. Cells growing in a log phase were treated with trypsin and re-suspended as single-cell solutions three days after transfection. Cells $\left(2.5 \times 10^{4}\right)$ were plated in each insert of BD BioCoat Matrigel invasion chambers (Becton-Dickinson, USA) with $500 \mu 1$ medium containing $0.1 \% \mathrm{BSA}$. In the lower chamber, $750 \mu \mathrm{l}$ of growth medium with $10 \%$ FBS was added as a chemoattractant. After incubation for $18 \mathrm{~h}$ at $37^{\circ} \mathrm{C}$ and $5 \% \mathrm{CO}_{2}$, the cells on the top surface of the insert were removed using a cotton swab. The migration assay was similar to the cell Matrigel invasion assay, except that the Transwell membrane was not pre-coated with ECMatrix and the cells were incubated for $16 \mathrm{~h}$ at $37^{\circ} \mathrm{C}$ and $5 \% \mathrm{CO}_{2}$. Cells adhering to the lower surface were fixed by methanol, stained by Wright-Giemsa staining, and counted under a microscope (x200). The invading cells on each set of triplicate membranes were counted. Means were based on the numbers from the triplicate wells for each treatment condition and were analyzed using a two-sided Student's t-test. Western blots were run to make sure cells were transfected in every experiment.

RNA extraction. Cells treated with siRNA-1 or with negative control siRNA were used for RNA extraction 3 days after transfection. Total RNA was prepared using an RNeasy mini kit (Qiagen, USA). Equal amounts of RNA were pooled and used for RT-PCR array analysis. RNA integrity was assessed spectrophotometrically; all samples had 260/280 $\mathrm{nm}$ ratios above 2.0 and $250 / 230 \mathrm{~nm}$ ratios above 1.7 . The quality of the purified RNA was assessed using an Agilent Bioanalyzer RNA 6000 Nano Assay.

Quantitative RT-PCR. Reverse transcription(RT) was performed using the ReactionReady First Strand cDNA Synthesis kit (SABiosciences, Frederick, USA). The polymerase chain reaction (PCR) was performed using the $\mathrm{RT}^{2}$ Profiler PCR Array PAHS-033C-2 and a Human Cancer Pathway Finder (SuperArray) on a Bio-Rad cycler with RT $^{2}$ Real-Time SYBR Green/ROX PCR Master mix PA-012. Five housekeeping 

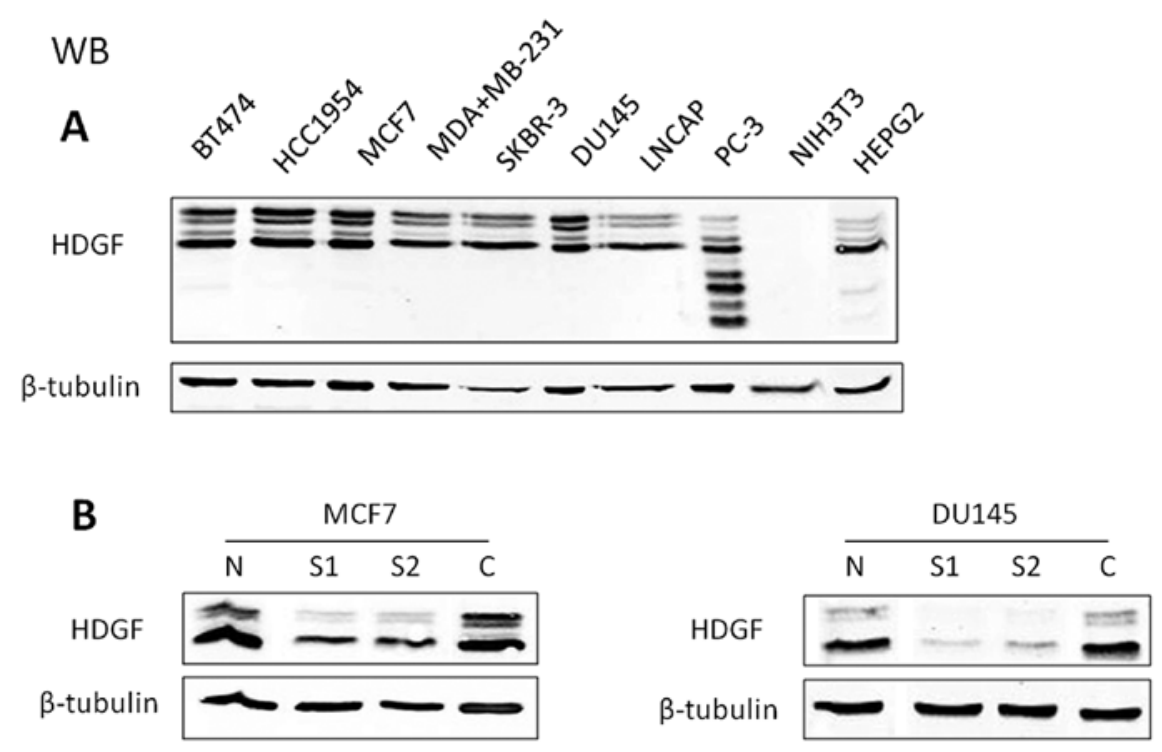

Figure 1. (A) Knockdown of HDGF in MCF7 and DU145 cells. HDGF was highly expressed in the prostate cancer cell line DU145 and in the breast cancer cell line MCF7. (B) DU145 and MCF7 were transfected with two HDGF siRNAs (S1 and S2). N, negative siRNA; C, transfection reagent only. Both siRNA treatments resulted in a decrease in HDGF expression.

genes, RT controls and PCR controls were included. A more detailed description of these procedures has been published (17). The array evaluated the expression of 84 genes involved in invasion and metastasis. Genes of the siRNA treatment group that differed by 1.5 -fold from negative controls and for which a $\mathrm{P}<0.05$ was obtained are emphasized. Additional genes for which fold-changes were between 1.1 and 1.5, but for which the P-value was also $<0.05$, are briefly noted.

Statistical analysis. The parametric Student's t-test was used to assess the significance of the differences between conditions of interest using SPSS 16.0 software. Values are expressed as the mean \pm standard deviation (SD) from at least three independent experiments in all analyses; a P-value of $<0.05$ was considered as statistically significant.

\section{Results}

siRNAs effectively knock down HDGF in breast cancer and prostate cancer cell lines. HDGF is highly expressed in breast cancer and prostate cancer cells lines (Fig. 1A). To investigate the involvement of HDGF in breast cancer and prostate cancer, HDGF expression was knocked down using siRNAs targeting the $H D G F$ gene. Western blot analyses showed that the HDGF protein levels were significantly reduced in the MCF7 and DU145 cell lines. siRNAs targeting HDGF suppressed the expression of HDGF at the protein level in breast and prostate cancer cells (Fig. 1B). siRNA-1 (S1) and siRNA-2 (S2) decreased HDGF protein by approximately $75 \%$ relative to the negative control (Fig. 1B), and were therefore used in subsequent experiments.

Knocking down HDGF expression inhibits cell growth. We next asked whether HDGF was functionally associated with malignant phenotypes of human cancers. Using RNA interference, the cell lines MCF7 and DU145, which express high levels of
HDGF, were treated with siRNA and their phenotypic changes were examined. Cell growth, as determined by the MTS assay and thymidine incorporation, showed significant suppression of proliferation in HDGF-siRNA-treated cells when compared with negative control siRNA-treated cells (Fig. 2). In the two cell lines, silencing of HDGF consistently resulted in a decrease of growth capability under culture conditions with or without serum, suggesting that HDGF is important for the proliferation of breast and prostate cancer cells.

HDGF expression knockdown reduces anchorage-independent growth. The effect of HDGF on anchorage-independent growth of DU145 and MCF7 cells was analyzed using the soft agar colony formation assay. Three weeks after seeding, cells transfected with HDGF-siRNA-1 produced significantly fewer colonies than cells transfected with negative control siRNA (Table I). These results suggest that HDGF is involved in anchorage-independent cell growth of DU145 and MCF7 cells, which is a feature of malignant transformation.

Knocking down HDGF expression suppressed migration and invasion by DU145 cells. To investigate whether HDGF is involved in prostate cancer migration and metastasis, we examined the in vitro invasive capacity of DU145 cells after HDGF knockdown. Transwell assays with Matrigel-coated filters revealed that the number of cells penetrating through the artificial basement membrane decreased significantly for HDGF-siRNA-treated cells ( $\mathrm{S} 1, \mathrm{P}=0.07$; $\mathrm{S} 2, \mathrm{P}=0.005$; Fig. 3). We also examined the migration capacity of HDGF knockdown DU145 cells in Transwell assays without Matrigel coating: the number of migrating cells also decreased significantly for HDGF-siRNA-treated DU145 cells ( $\mathrm{S} 1, \mathrm{P}=0.06$; $\mathrm{S} 2, \mathrm{P}=0.01$; Fig. 4). These results, along with the reduced proliferation rate in HDGF knockdown clones described above, suggest that HDGF may be involved in tumor growth and metastasis in prostate cancer. 
A
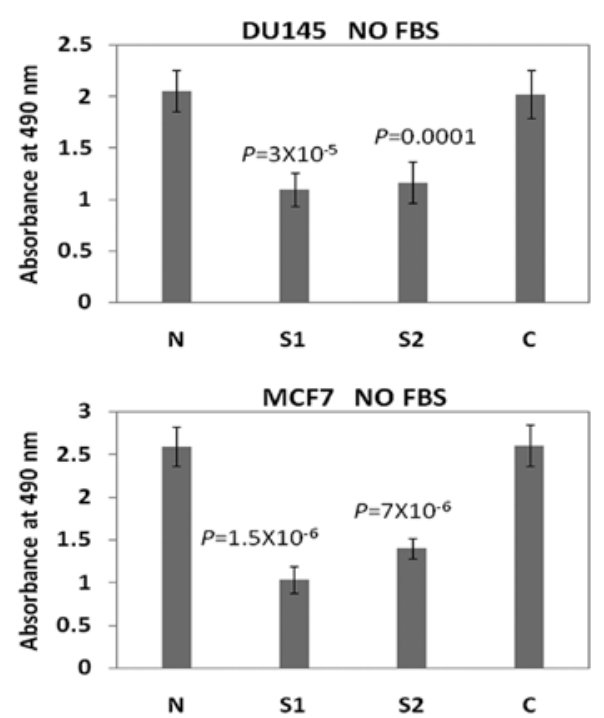

B Thymidine Incorporation Assay
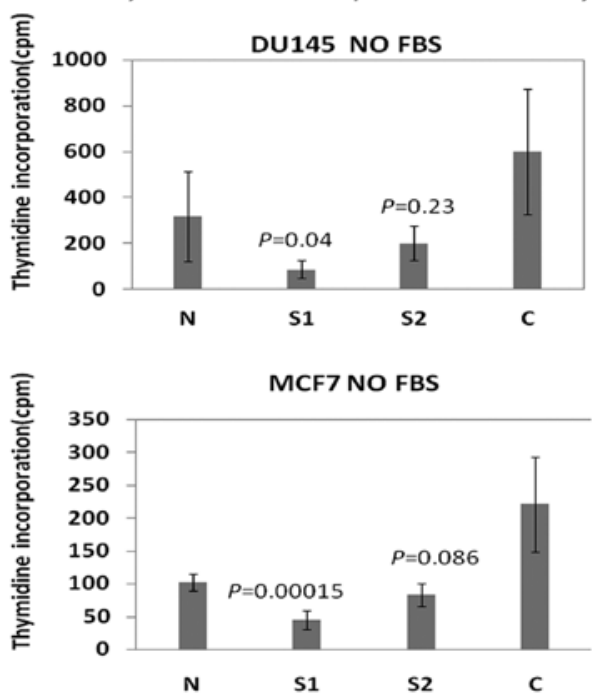
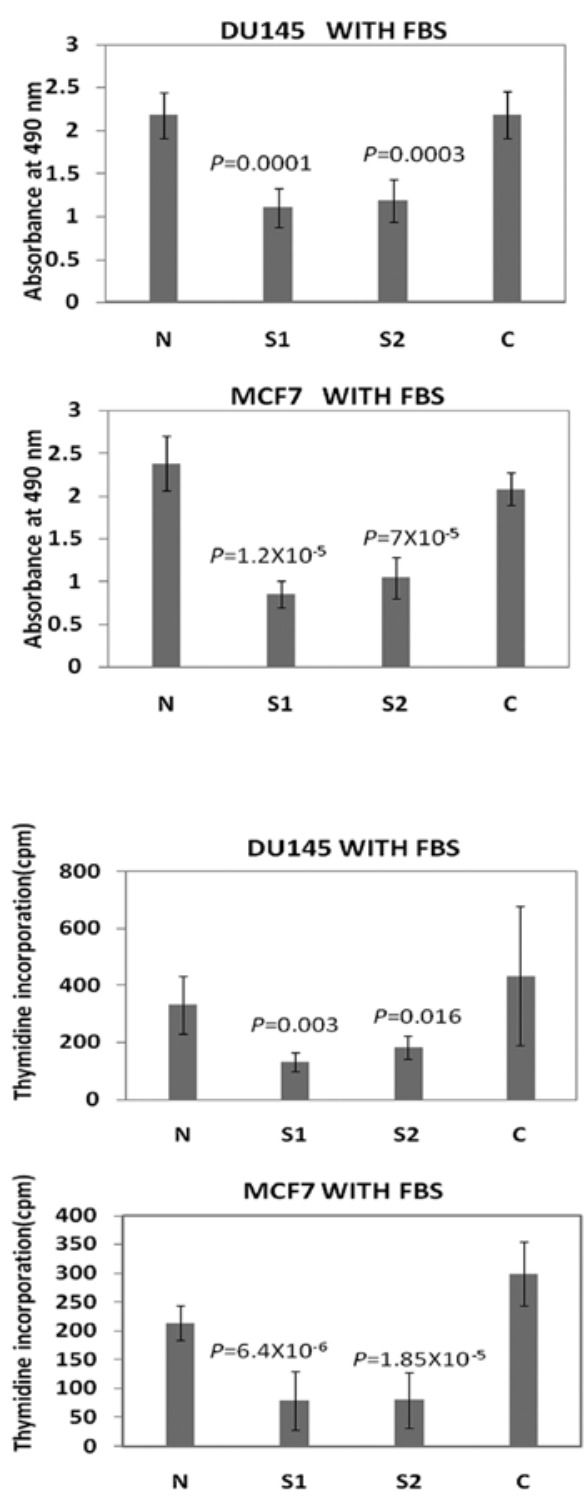

Figure 2. Down-regulation of HDGF suppressed the growth of DU145 and MCF7 cells. (A) After 5 days of transfection, the cells were examined using the MTS assay. (B) After 5 days of transfection, the cells were examined using the thymidine incorporation assay. Error bars: the standard deviation from five separate experiments. The protein expression level of HDGF in cells after transfection was confirmed with Western blots for each experiment. Compared with cells in the negative control group $(\mathrm{N})$, the growth of siRNA-treated $(\mathrm{S} 1, \mathrm{~S} 2)$ cells significantly decreased $\left({ }^{*} \mathrm{P}<0.05\right)$.

Table I. Effect of HDGF down-regulation on anchorage-independent cell growth. ${ }^{a}$

\begin{tabular}{lccrc}
\hline Cell line & N & S1 & C & P-value (N vs. S1) \\
\hline DU145 & $436.33 \pm 12.10$ & $165.33 \pm 23.07$ & $452 \pm 35.04$ & 0.0054 \\
MCF7 & $969.66 \pm 50.33$ & $240.00 \pm 19.97$ & $1189.66 \pm 91.22$ & 0.0016
\end{tabular}

${ }^{a}$ Number of colonies visible in a microscope field at $\mathrm{x} 40$ magnification; the value is the average of triplicate wells and four randomly selected fields per well. N, negative siRNA control group; S1, siRNA-1-treated; C, transfection reagent only group.

Knocking down HDGF expression stimulated migration and invasion in MCF7 cells. We also examined the effects of HDGF knockdown on the invasive capacity of MCF7 cells. Chamber assays using Matrigel-coated filters revealed that the number of MCF7 cells penetrating through the artificial basement membrane increased significantly in HDGF-siRNAtreated cells relative to negative control cells $(\mathrm{S} 1, \mathrm{P}=0.0004$; $\mathrm{S} 2, \mathrm{P}=0.001$; Fig. 3). In the migration assays, the number of 
Invasion Assay

$\mathrm{N}$
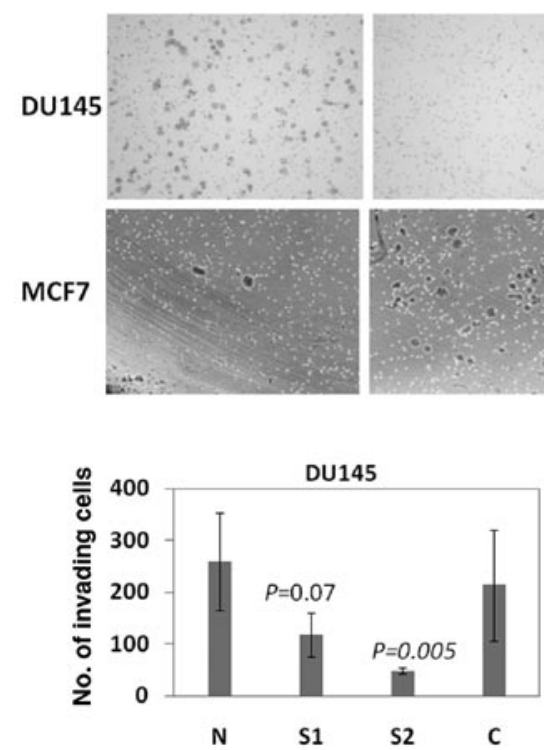

S1

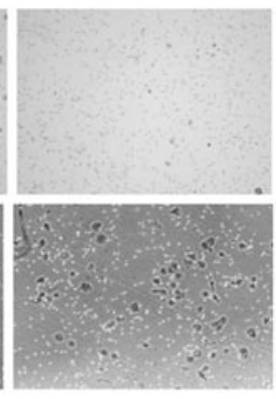

S2

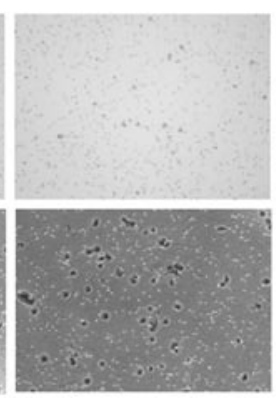

C

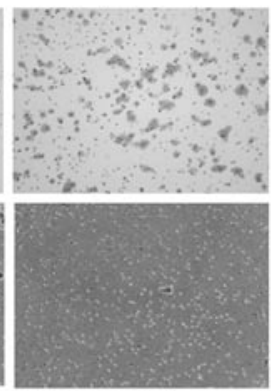

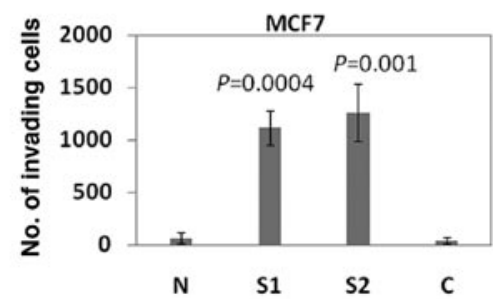

Figure 3. Effect of knockdown of HDGF on DU145 and MCF7 cell invasion. A chamber invasion assay of DU145 and MCF7 cells with negative siRNA (N), siRNA-1 (S1), siRNA-2 (S2) and transfection reagent only (C). At $72 \mathrm{~h}$ after transfection, invaded cells were fixed, stained and counted. siRNA-treated DU145 cells showed significantly decreased invasion. siRNA-treated MCF7 cells showed significantly increased invasion. Representative photos show migrated cells at X100 magnification. In the graphs, bars represent the mean number of cells; error bars represent SD; P-values are relative to the negative control.

\section{Migration Assay}
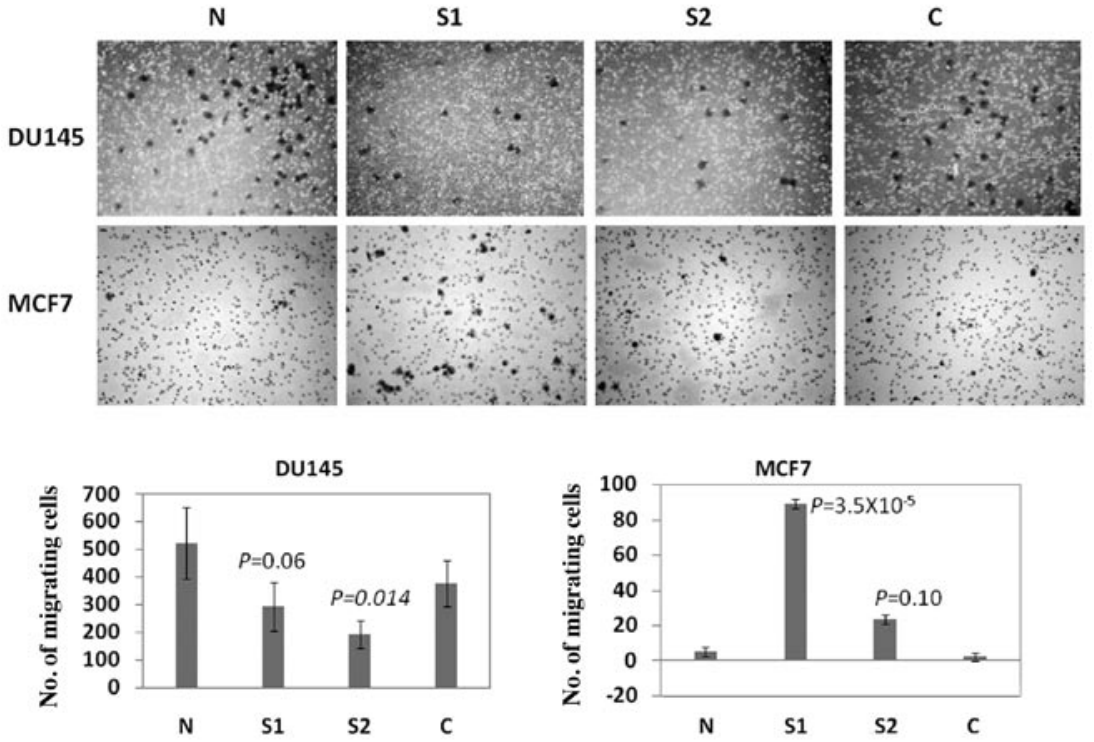

Figure 4. Effect of HDGF knockdown on DU145 and MCF7 cell migration. A Transwell migration assay on DU145 and MCF7 cells with negative siRNA (N), siRNA-1 (S1), siRNA-2 (S2), transfection reagent only (C). After $72 \mathrm{~h}$ of transfection, migrated cells were fixed, stained and counted. siRNA-treated DU145 cells showed significantly decreased cell migration. siRNA-treated MCF7 cells had significantly increased cell migration. The representative photographs show migrated cells at X100 magnification). In the graphs, bars represent the mean number of cells; error bars represent SD; the P-values are relative to the negative control.

migrating HDGF-siRNA-treated MCF7 cells also increased significantly relative to the number of negative control cells (S1, P=3.5x10-5; S2, P=0.10; Fig. 4).

The results of these experiments suggest that HDGF was required for cell growth, while inhibiting invasion by MCF7 cells. It is intriguing to anticipate the underlying mechanism(s) of how HDGF knockdown induces opposite effects on cell invasion in DU145 and MCF7 cells.

PIK3RI and SERPINE1 are differentially expressed under HDGF-siRNA-1 treatment. To explore how HDGF knockdown induced various effects on cell proliferation, migration, and 
qRT-PCR
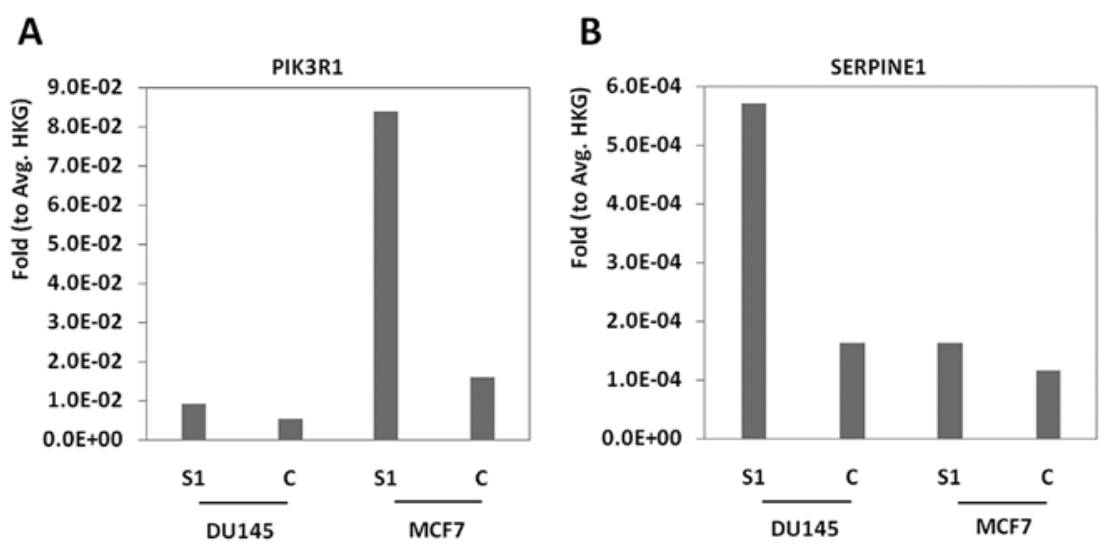

Figure 5. PIK3R1 and SERPINE1 mRNA expression regulation in MCF7 and DU145 cell lines in response to HDGF siRNA treatment. mRNA expression of PIK3RI (A) and SERPINE1 (B) before and after treatment with control siRNA or with $10 \mathrm{nmol} / 1$ HDGF-siRNA-1 in DU145 and MCF7 cells as measured by qRT-PCR array analysis. HKG, housekeeping gene.

invasion, we used RT-PCR to measure the expression of 84 genes implicated in tumor growth and metastasis. We compared gene expression profiles between cells treated with negative control siRNA and cells treated with 10 nM HDGF-siRNA-1 at the 72-h time-point. In HDGF-siRNA-1-treated DU145 cells, the SERPINE1 gene was up-regulated $\geq 3$-fold compared with cells treated with control siRNA. In HDGF-siRNA-1treated MCF7 cells, the PIK3R1 gene was up-regulated $\geq 5$-fold compared with cells treated with control siRNA (Fig. 5). None of the genes was down-regulated $>3$-fold. PIK3RI has been implicated in the migration and invasion of MCF7 cells (18). SERPINE1 encodes plasminogen activator inhibitor-1 (PAI-1), inhibitor of PLAU and PLAUR, which plays an important role in the migration and invasion of prostate cancer cells (19).

\section{Discussion}

$H D G F$ encodes a member of the hepatoma-derived growth factor family. The protein has a wide range of biological functions in cellular biology, such as mitogenic activity (5), promotion of angiogenesis (20) and lung remodeling (21). Clinical studies have shown that overexpression of HDGF is associated with poor outcomes of tumors such as gastric cancer (22), lung cancer (11-13), liver cancer $(23,24)$, colorectal stromal tumors (25) and pancreatic cancer (15) in humans. HDGF expression significantly correlates with VEGF expression and is a valuable prognostic factor for human hilar cholangiocarcinoma (26). In agreement with these studies, we observed high levels of HDGF expression in prostate and breast cancer cell lines. This indicated that HDGF may play a role in the development of breast and prostate cancer.

In this study, we knocked down HDGF in prostate and breast cancer cells using siRNA targeting HDGF. HDGF knockdown consistently inhibited proliferation in both types of cells. However, HDGF knockdown inhibited migration and invasion by prostate DU145 cells, but it stimulated migration and invasion by breast MCF7 cells. This effect may result from the differential induction of PIK3RI and SERPINE1 in the two cell lines. Therefore, the present study provides the first evidence supporting the role of HDGF expression during the proliferation of prostate cancer and breast cancer cells.

In our study, we observed that knockdown of HDGF expression by siRNA in DU145 cells could markedly decrease cell proliferation, as well as migration and invasion capacity. Down-regulation of HDGF also suppressed proliferation of MCF7 cells, but it stimulated their migration and invasion capacity. MCF7 cells display epithelial morphology and very weak invasive capability. We observed an EMT-like phenotypic change in MCF7 cells following HDGF knockdown (data not shown). DU145 cells show a heterogeneous morphology resembling both epithelial and mesenchymal cells; therefore, the invasive activity of DU145 cells is much stronger than that of MCF7 cells. This difference in phenotypic features and genetic background may result in different signaling pathways being related to HDGF. The molecular mechanisms underlying the HDGF contribution to the proliferation and invasive capacity of DU145 cells remain to be explored. Future studies of HDGF expression in vivo may be justified in order to extrapolate our findings to a clinical setting. Our results provide insight into the involvement of HDGF in the development of prostate cancer and breast cancer cells. HDGF is a potential target for prostate cancer.

Down-regulation of HDGF can lead to up-regulation of the pro-apoptotic protein Bad and to inactivation of ERK and Akt. The latter, in turn, leads to dephosphorylation of Bad and activation of the intrinsic apoptotic pathway $(27,28)$. Enforced expression of HDGF could inhibit nordihydroguaiaretic acid (NDGA)-induced apoptosis through the mitochondrial pathway in colorectal cancer cells and attenuate the inhibitory effect of NDGA on tumor growth. Therefore, HDGF is a molecule potentially associated with chemotherapy resistance (29). It will be valuable to determine whether HDGF contributes to the development of prostate cancer using similar pathways. HDGF stimulates cell proliferation by functioning on both sides of the plasma membrane, as a ligand for membrane receptor binding that triggers cell signaling and as a stimulator for DNA synthesis within the nucleus. HDGF exploits the innate properties of both cell surface heparan sulfates (HS) 
and membrane receptors via the HATH domain to affect related cell signaling processes. MAPK signaling pathways could be affected by the HS-mediated HATH internalization to regulate the migration of NIH3T3 fibroblasts, as judged from the differential effect of treatment with HATH and the HATH(K96A) mutant form on the expression level of matrix metalloproteinases (30).

In conclusion, HDGF may play a key role in promoting the tumor growth of DU145 and MCF7 cells. HDGF is involved in anchorage-independent growth and invasion by breast and prostate cancer cells. These qualities may contribute to the HDGF-associated aggressive biological behavior of such cancers. The mechanism of HDGF-mediated aggressiveness in cells needs to be further elucidated.

\section{Acknowledgements}

This study was supported by the Jay and Betty Van Andel Foundation. We thank Mr. David Nadziejka at VARI for technical editing of the manuscript and Ms. Amy Nelson for administrative assistance.

\section{References}

1. Nakamura H, Kambe H, Egawa T, et al: Partial purification and characterization of hepatoma-derived growth factor. Clin Chim Acta 183: 273-284, 1989.

2. Nakamura H, Izumoto Y, Kambe H, et al: Molecular cloning of complementary DNA for a novel human hepatoma-derived growth factor. Its homology with high mobility group-1 protein. J Biol Chem 269: 25143-25149, 1994.

3. Okuda Y, Nakamura H, Yoshida K, et al: Hepatoma-derived growth factor induces tumorigenesis in vivo through both direct angiogenic activity and induction of vascular endothelial growth factor. Cancer Sci 94: 1034-1041, 2003.

4. Izumoto Y, Kuroda T, Harada H, Kishimoto T and Nakamura H: Hepatoma-derived growth factor belongs to a gene family in mice showing significant homology in the amino acid terminus. Biochem Biophys Res Commun 238: 26-32, 1997.

5. Kishima Y, Yamamoto H, Izumoto Y, et al: Hepatoma-derived growth factor stimulates cell growth after translocation to the nucleus by nuclear localization signals. J Biol Chem 277 10315-10322, 2002.

6. Everett AD, Stoops TD and McNamara CA: Nuclear targeting is required for hepatoma-derived growth factor stimulated mitogenesis in vascular smooth muscle cells. J Biol Chem 276 37564-37568, 2001.

7. Oliver JA and Al-Awqati Q: An endothelial growth factor involved in rat renal development. J Clin Invest 102: 1208-1219, 1998.

8. Everett AD, Lobe DR, Matsumura ME, Nakamura $H$ and McNamara CA: Hepatoma-derived growth factor stimulates smooth muscle cell growth and is expressed in vascular development. J Clin Invest 105: 567-575, 2000.

9. Enomoto H, Yoshida K, Kishima Y, et al: Hepatoma-derived growth factor is highly expressed in developing liver and promotes fetal hepatocyte proliferation. Hepatology 36: 1519-1527, 2002.

10. Kishima Y, Yoshida K, Enomoto H, et al: Antisense oligonucleotides of hepatoma-derived growth factor (HDGF) suppress the proliferation of hepatoma cells. Hepatogastroenterology 49: 1639-1644, 2002.

11. Zhou SJ, Xu SF, Zhang HQ and Liu ZD: Expression of HDGF and its implication in stage I non-small cell lung cancer. Zhonghua Zhong Liu Za Zhi 29: 927-930, 2007.
12. Iwasaki T, Nakagawa K, Nakamura H, Takada Y, Matsui K and Kawahara K: Hepatoma-derived growth factor as a prognostic marker in completely resected non-small cell lung cancer. Oncol Rep 13: 1075-1080, 2005.

13. Ren H, Tang X, Lee JJ, et al: Expression of hepatoma-derived growth factor is a strong prognostic predictor for patients with early-stage non-small cell lung cancer. J Clin Oncol 22: 3230-3237, 2004.

14. Zhang J, Ren H, Yuan P, Lang W, Zhang L and Mao L: Downregulation of hepatoma-derived growth factor inhibits anchorage-independent growth and invasion of non-small cell lung cancer cells. Cancer Res 66: 18-23, 2006.

15. Uyama H, Tomita Y, Nakamura H, Nakamori S, Zhang BL and Hoshida Y: Hepatoma-derived growth factor is a novel prognostic factor for patients with pancreatic cancer. Clin Cancer Res 12: 6043-6048, 2006.

16. Zhang A, Long W, Guo Z, et al: Development and clinical evaluation of a multi-purpose $\mathrm{mAb}$ and a sandwich ELISA test for hepatoma-derived growth factor in lung cancer patients. J Immunol Methods 15: 61-67, 2010.

17. Gridley DS, Slater J, Luo-Owen X, et al: Spaceflight effects on T lymphocyte distribution, function and gene expression. J Appl Physiol 106: 194-202, 2009.

18. Uchino M, Kojima H, Wada K, et al: Nuclear beta-catenin and CD44 upregulation characterize invasive cell populations in non-aggressive MCF-7 breast cancer cells. BMC Cancer 10: 414, 2010.

19. Ashida S, Nakagawa H, Katagiri T, et al: Molecular features of the transition from prostatic intraepithelial neoplasia (PIN) to prostate cancer: genome-wide gene-expression profiles of prostate cancers and PINs. Cancer Res 64: 5963-5972, 2004.

20. Everett AD, Narron JV, Stoops T, Nakamura H and Tucker A: Hepatoma-derived growth factor is a pulmonary endothelial cell-expressed angiogenic factor. Am J Physiol Lung Cell Mol Physiol 286: L1194-L1201, 2004.

21. Mori M, Morishita H, Nakamura H, et al: Hepatoma-derived growth factor is involved in lung remodeling by stimulating epithelial growth. Am J Respir Cell Mol Biol 30: 459-469, 2004.

22. Yamamoto S, Tomita Y, Hoshida Y, et al: Expression of hepatoma-derived growth factor is correlated with lymph node metastasis and prognosis of gastric carcinoma. Clin Cancer Res 12: $117-122,2006$.

23. Yoshida K, Tomita Y, Okuda Y, et al: Hepatoma-derived growth factor is a novel prognostic factor for hepatocellular carcinoma. Ann Surg Oncol 13: 159-167, 2006.

24. Zhou Y, Zhou N, Fang W and Huo J: Overexpressed HDGF as an independent prognostic factor is involved in poor prognosis in Chinese patients with liver cancer. Diagn Pathol 5: 58, 2010.

25. Hu TH, Lin JW, Chen HH, Liu LF, Chuah SK and Tai MH: The expression and prognostic role of hepatoma-derived growth factor in colorectal stromal tumors. Dis Colon Rectum 52: 319-326, 2009.

26. Liu YF, Zhao R, Guo S, Wang XQ, Lian PL, Chen YG and $\mathrm{Xu} \mathrm{KS}$ : Expression and clinical significance of hepatomaderived growth factor as a prognostic factor in human hilar cholangiocarcinoma. Ann Surg Oncol 18: 872-879, 2011.

27. Mao J, Xu Z, Fang Y, et al: Hepatoma-derived growth factor involved in the carcinogenesis of gastric epithelial cells through promotion of cell proliferation by Erk1/2 activation. Cancer Sci 99: 2120-2127, 2008

28. Tsang TY, Tang WY, Tsang WP, Co NN, Kong SK and Kwok TT: Down-regulation of hepatoma-derived growth factor activates the Bad-mediated apoptotic pathway in human cancer cells. Apoptosis 13: 1135-1147, 2008.

29. Liao F, Liu M, Lv L and Dong W: Hepatoma-derived growth factor promotes the resistance to anti-tumor effects of nordihydroguaiaretic acid in colorectal cancer cells. Eur J Pharmacol 645: 55-62, 2010.

30. Wang CH, Davamani F, Sue SC, et al: Cell surface heparan sulfates mediated internalization of PWWP/HATH domain of HDGF via macropinocytosis to fine-tune cell signaling process involved in and its effect on fibroblast cell migration. Biochem $\mathrm{J}$ 433: 127-138, 2010. 